\title{
Transforming Energy Making Use of Pyroelectric: A Conservation Technique
}

\author{
Shivam Dewan and Paras Arya
}

\begin{abstract}
A lot of energy around us gets wasted, keeping in mind, the increasing energy demand and scarcity of resources, we have devised an idea to conserve the heat energy, which gets emitted out by the refrigerator. The technique works as follows : a pyrolectric transducer is attached to the compressor pump of the refrigerator, aligned in such a way that one side of it is line other contact with the compressor and other with the wall( ceiling ) or the other part is at room temperature. This temperature of the compressor is approx. 50-75 celsius higher than the room temperature. This creates a temperature difference between the two sided of the transducer which in turn modifies the orientation of the atoms, leading to the change in polarization and thus giving rise to a constant voltage across the transducer(pyroelectric).This transducer is further connected to a capacitor. Due to this voltage across the transducer, the capacitor gets charged up and this can be further used as a supply to any appliance (operating at low voltage).
\end{abstract}

Index Terms-Capacitor, compressor, pyroelectric, sustainable energy.

\section{INTRODUCTION}

The heat energy is emitted by the compressor of the refrigerator which leads to an increase in its temperature $(d T)$. dt. Here, denotes the change in the temperature. The compressor is further attached to the pyroelectric substance [1]. A pyroelectric substance when experience a temperature change that generates voltage across it [2]. This temperature change is sensed by the pyro electric, thus generating a voltage across it. The Voltage is generated only if $d T / d t>0$ or $d T / d t<0(d T / d t$ represents the change in temperature with respect to time), and no voltage is observed when $d T / d t=0$. In order to utilize this voltage across the pyroelectric substance [1], the setup is further connected in the capacitor. Since, the connection is in parallel, the same voltage drop occurs across the capacitor [3]. Now, this charged capacitor can be used for small voltage rating appliances.

\section{COMPRESSOR}

The refrigerator compressor is both a motor and a pump that move the refrigerant through the system. Temperature sensors signal the compressor to start when the temperature inside the refrigerator rises above its set point [4]. No

Manuscript received September 25, 2013; revised April 27, 2014.

Shivam Dewan is with the Northern India Engineering College, Guru Gobind Singh Indraprastha University, Delhi, India (e-mail: dewan.shivam123@yahoo.com).

Paras Arya was with Guru Gobind Singh Indraprastha University, Delhi India (e-mail: parasarya92@yahoo.in). refrigerator is completely airtight; cold air leaks out, and warmer air leaks in, causing the temperature to rise above its set point. As the compressor starts, it draws in the cold refrigerant gas in liquid form as it leaves the evaporator [5]. Refrigerators use a type of refrigerant gas that turns into a liquid at very cold temperatures. The compressor then puts pressure on the gas---compressing it. As the gas is compressed, its temperature goes up. The compressor pushes out the hot, compressed gas through the outside metal coils (tubes) on the back or bottom of the refrigerator. These coils allow the heat to dissipate into the surrounding air. Because it is under pressure, the gas changes into a liquid as it cools. The liquid gas continues to flow through the system until it reaches the expansion valve. The valve forces the liquid through a very small hole, turning it into a very cold mist, which evaporates as it moves through the freezer coils. As the cold liquid gas moves through the coils, it draws heat out of the surrounding air in the freezer and refrigerator compartments. This is based on the second law of thermodynamics: heat moves from warmer objects to colder objects. The colder object is the evaporating gas, and the warmer object is the air. The coils lead back to the compressor [4]. When the liquid reaches the compressor, pressure is applied. The compressor sends the hot gas back through the outside coils to release heat into the air. The process of compression and evaporation continues until the refrigerator temperature has returned to its set point. Once the temperature is at its set point, the temperature sensors signal the compressor to stop.

As per the observations the temperature of the compressor reaches to a value approximately $50-75^{*} \mathrm{C}$ higher than the room temperature [6]. The compressor works on the second law of the thermodynamics. After the set point is reached, the compressor stops working.

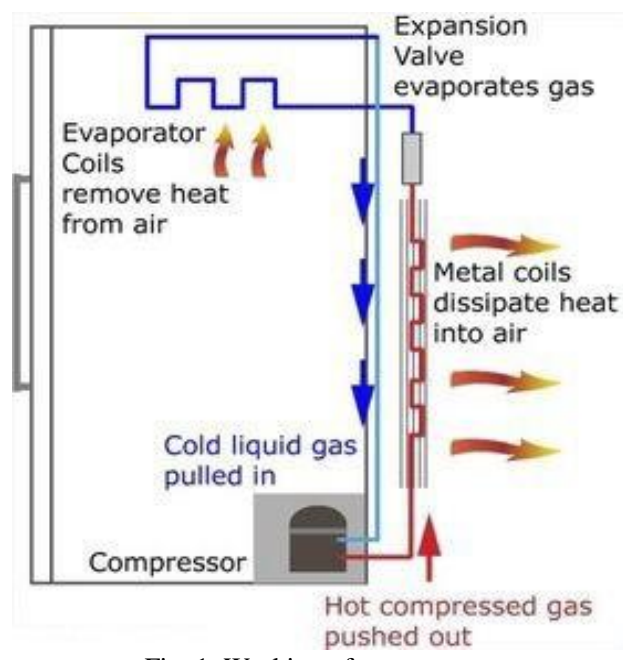

Fig. 1. Working of compressor. 
The Fig. 1 here completely depicts the working of the refrigerator. The evaporator coils remove heat from the air, thus expansion valve evaporates gas, cold liquid being pulled in the compressor and finally the metal coils dissipate heat to the outside surroundings.

\section{Voltage Generation USING -PyroeleCtrics}

Pyrolectrics are those materials which when experience a temperature difference, either heated or cooled, give rise to a voltage across them [7]. The cyrstal structure of the pyroelectric experiences a modification of the atoms. Within their crystal structure, the modification of the atoms leads to the change in its polarisation thus producing a small voltage. The change in polarisation vector with respect to the temperature gives rise to this voltage. The seminal advantage of using a pyroelectric is that it can be repearedky cooled or heated to generate electrical power [8].

Where, $P i\left(\mathrm{~cm}^{-2} K^{-1}\right)$ is the vector for the pyroelectric coefficient.

Spontaneous polarization is temperature dependent, so a good perturbation probe is a change in temperature which induces a flow of charge to and from the surfaces. All polar crystals are pyroelectric [6], so the 10 polar crystal classes are sometimes referred to as the pyroelectric classes. The property of pyroelectric crystal is to measure change in net polarization (a vector) proportional to a change in temperature. The total pyroelectric coefficient measured at constant stress is the sum of the pyroelectric coefficients at constant strain (primary pyroelectric effect) and the piezoelectric contribution from thermal expansion (secondary pyroelectric effect).

By this equation, the volatge across the pyrolectrics is given a change in polarization with respect to the temperatre [9]. The diagram illustrates the pyroelectric material's change in the orientation with temperaure (with respect to time).

When there is no temperature difference, that is, the compressor is not working, then the ammeter does not experience any deflection as shown in Fig. 2(a) [10]. But when the temperature change with respect to time is positive, the ammeter shows a negative deflection, Fig. 2(b). Similarly, the negative change across the pyroelectric deviates the ammeter's reading to the right, Fig. 2(c).

As per the observation, the temperature of the compressor reaches a value $50-75 \times \mathrm{C}$ above the room temperature, one side of the pyrolectric is at room temperature, whilst the otherat a temperature approx. $60 \times \mathrm{C}$ higher. Thus, a negative volatge drop is observed across the pyroelectric materials [11], negative volage drop signifies that charges are flowing from the earh( taken as reference) to the pyroelctric [12]. Pyroelectricity relates a change in temperature to a change in electrical displacement $D\left(C / \mathrm{m}^{2}\right) d D=p d T$-(1) (Where $p$ is pyroelectric coefficeint $\left(C / \mathrm{m}^{2} K\right), d T$ represents change in temperature).

The equation for current is given by : $I=p A(d T / d t)-(2)$ (Where, $p$ is the pyroelectric coefficeint, $\mathrm{A}$ is the electrode area, and $d T / d t$ is the rate of change in temperature.) According to this equation, the current will linearly increase with increasing the rate in the temperature.
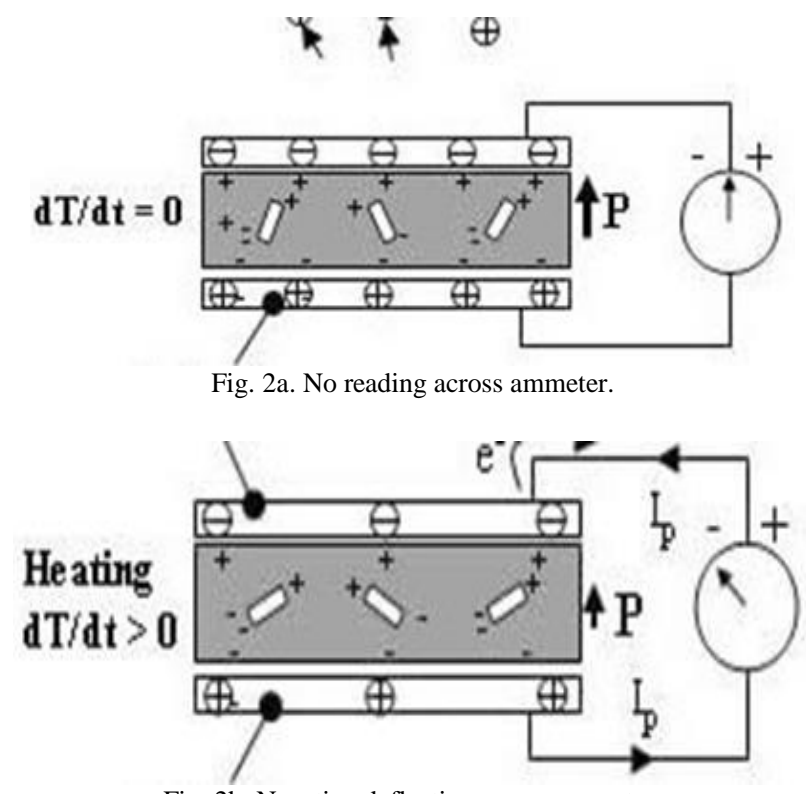

Fig. 2b. Negative deflection across ammeter.

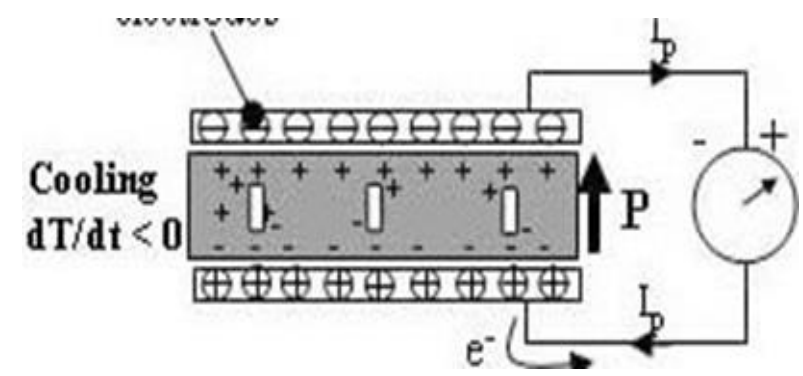

Fig. 2c. Positive deflection across ammeter.

Pyroelectric materials based on lead zirconatetitanate (PZT) film, which has pyroelectric cofficient of about$80 \mathrm{nC} / \mathrm{cm}^{2} \mathrm{~K}$. For a temperature change of $45 \mathrm{~K}$ at a rate of $0.2 \mathrm{~K} / \mathrm{s}$, the output open circuit voltage and short-circuit current density of the pyroelectric reached $22 \mathrm{~V}$ and $171 \mathrm{nA} / \mathrm{cm}^{2}$.

The setup is further connected to a capacitor. A Capacitor is a passive device that stores energy in its Electric Field and returns energy to the circuit whenever required. A Capacitor consists of two Conducting Plates separated by an Insulating Material or Dielectric.

When a Capacitor is connected to a circuit with Direct Current (DC) source, two processes, which are called "charging" and "discharging" the Capacitor, will happen in specific conditions.

In Fig. 3, the Capacitor is connected to the DC Power Supply and Current flows through the circuit. Both Plates get the equal and opposite charges and an increasing Potential Difference, $v_{c}$, is created while the Capacitor is charging. Once the Voltage at the terminals of the Capacitor, $v_{c}$, is equal to the Power Supply Voltage, $v_{c}=V$, the Capacitor is fully charged and the Current stops flowing through the circuit, the Charging Phase is over.

Since the capacitor is in parallel connection as a result the same pyroelectric voltage will build across the capacitor. The plates of the capacitor stores the charges in the electric field built across the capacitor plates. The charging of the capacitor is given in terms of the time constant $R C$ [13]. The equation which describes the charging of the capacitor is given by eq. (3). 
$Q(t)=C V\left[1-e^{-t / T]}\right.$

$(T(\mathrm{tau})=R C$

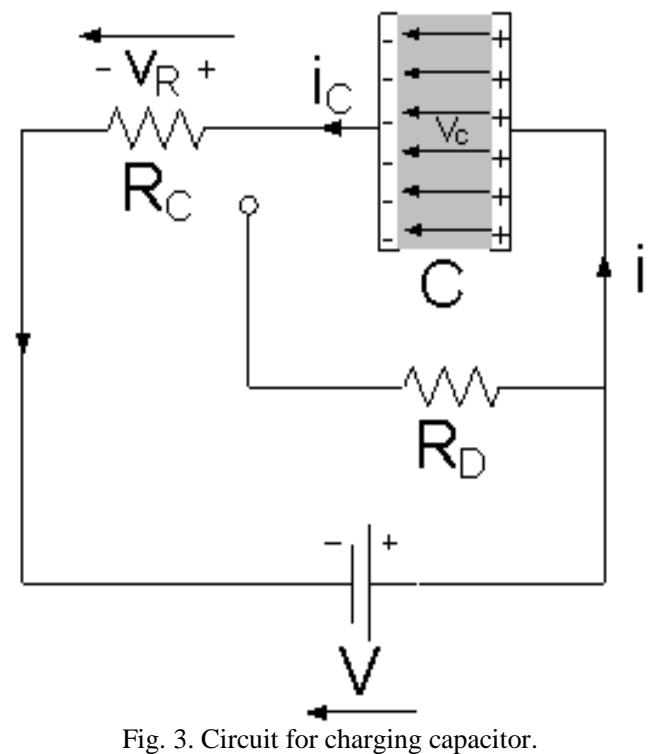

$Q(t)$ is the charge on the plates of the capacitor, $C$ is the capacitance and $\mathrm{RC}$ is the time constant.

As soon as the capacitor experiences this voltage drop, current flows and the potential difference across the capacitor begins to rise, but as more and more charge builds up on the capacitor plates, the current and the rate of rise of potential difference both fall. Finally no further current will flow when the potential difference across the capacitor equals that of supply voltage $V_{0}$. The capacitor is then fully charged [14]. The compressor when stops working the potential difference across the capacitor drops. As charge flows from one plate to the other the charge is neutralised and so the current falls and the rate of decrease of potential difference also falls [15].

Eventually the charge on the plates is zero and the current and potential difference are also zero, the capacitor is fully discharged [16]. Fig. 4 here shows the phenomena of charging of the capacitor.

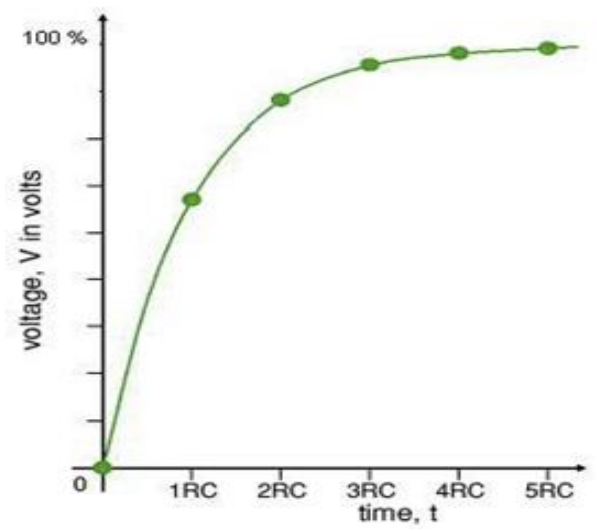

Fig. 4. charging of the capcitor.

A capacitor is equivalent to an open-circuit to direct current, $R=\infty$, because once the charging phase has finished, no more current flows through it. The voltage $v_{c}$ on a capacitor cannot change abruptly.

When the capacitor disconnected from the power supply, the capacitor is discharging through the resistor $R_{D}$ and the voltage between the plates drops down gradually to zero, $v_{c}=0$, Fig. 5 here depicts the discharging of capacitor.

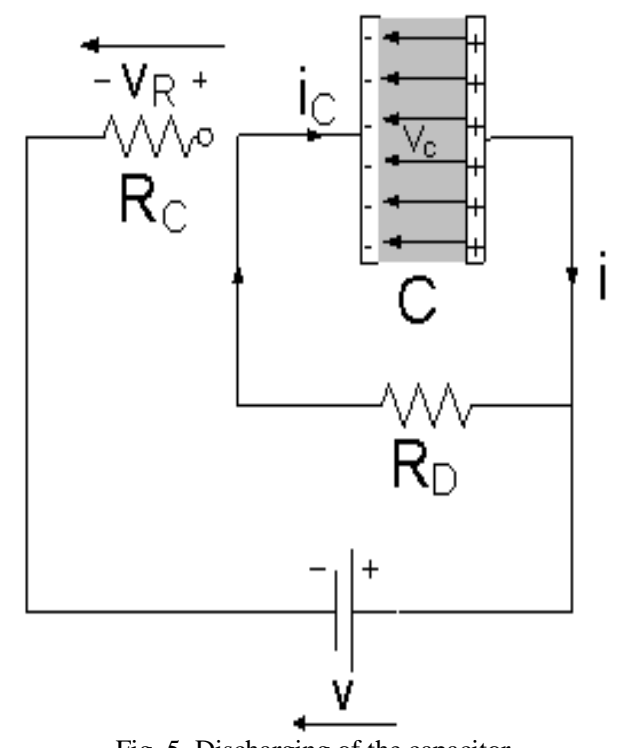

Fig. 5. Discharging of the capacitor.

\section{CONCLUSION}

When the compressor gets started its temperature rises. This rise in temperature across the compressor of the refrigerator introduces a voltage across the capacitor $(d T / d t<0)$, but it stays at this value of temperature (till it is working) and thus capacitor gets discharged $(d T / d t=0)$ in this interval. But when the set point inside the refrigerator is reached and compressor stops working and starts getting cooled up $(d T / d t<0)$, again generating a positive voltage value across the capacitor. This capacitor can further be used as a source for devices operating at small voltages.

\section{REFERENCES}

[1] A. K. Batra and M. Das, "Pyroelctric materails: nature, infrared rays, and energy harvester," vol. 1, pp. 197-255, 2013.

[2] A. Ford and R. Singh, "Science of conservable enery and particle accelerator," vol. 2, pp. 455-500, 2010.

[3] S. B. Lang, "Pyroelectricity: from ancient curiosity to modern imaging too," Physics Today, vol. 58, no. 8, pp. 31-36, 2005.

[4] X. Li, M. Watt, S. Lui, and A. Rosch, "Pyroelectrics and electrocarolic materials," vol. 1, 2006.

[5] M. Plakat, S. Bansal, and L. Ben, "Looking in sustainable development through peizo and pyrolectrics shells and energy harvesting," vol. 1, pp. 120-200, 2011.

[6] J. Jane, C. Merkel, and P. Lasm, "Developmental status through renewable and sustainable energies," vol. 2, pp. 88-174, 2002.

[7] Iana, J. Mueller, and I. Johana, "Pyroelectris: potential and capacity," vol. 2, pp. 52-122, 2004.

[8] X. Li and R. Khatar, "Waves in the pyroelectrics," vol. 2-vol. 3, pp. 122-199, 2005.

[9] T. Francis, "The inhomogenuous wave in the pyroelectrics," vol. 3, pp. $110-155,2000$

[10] J. Brown, E. Height, and L. Schnell, "Science of energy harvesting in the global world," vol. 1, pp. 88-220, 2003.

[11] S. B. Gupta, Y. Jang, and M. Jorkhani, "Structuring renewable enery through manifesting solar and peizoelectrics techniques," vol. 1, 1999.

[12] Y. Yang et al. "Pyroelectricnanogenerators fro driving wireless sensors," vol. 2, pp. 55-144, 2010.

[13] L. Zepone, and C. Bone, "Light of sustainable energies in terms of piezoelectric material," vol. 1, pp. 222-320.

[14] L. Names and J. Stunner, "Astounding facts od peizoelectrics and pyroelectrics materila leading to energy harvesting in field of developing countries," vol. 1, pp. 255-299, 2002. 
[15] Y. Siengh and P. Verma, "Future of development lies in sustainable funding of the assesment in sceince ofpyroelectrics," vol. 2, pp. 155200, 2004.

[16] S. Sigh and J. Wilson, "Pyroelectrics response od ferro electric nanowires," vol. 2, 2002.

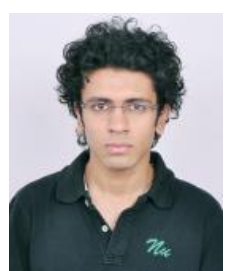

Shivam Dewan was born on $8^{\text {th }}$ April, 1992 in Delhi. He is currently persuing bachelor's degree in technology in mechanical and automation from Northern India Engineering College, Guru Gobind Singh Indraprastha University, Delhi, India.

$\mathrm{He}$ reached in the final round of the National Go Cart, Kolhapur, 2013.

$\mathrm{He}$ has done his summer training in
Manufacturing and Transmission Department, Escorts Group, Delhi. His research areas include sustainable and renewable energy stratergy, especially solar enery.

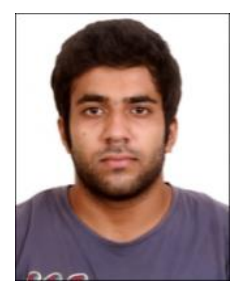

Paras Arya was born on $20^{\text {th }}$ November, $1992 . \mathrm{He}$ is currently persuing his bachelor's degree in technology in electronics and communication from Bhagwan Parshuram Institute of Technology affiliated to Guru Gobind Singh Indraprastha University, Delhi.

$\mathrm{He}$ did his summer training in Solar Cell Department from Central Electronics Limited, Sahibabad. He made his final year project named Temperature Sensing with Application Using Gsm Technology. 